\title{
Archival Report
}

\section{Exome Chip Meta-analysis Fine Maps Causal Variants and Elucidates the Genetic Architecture of Rare Coding Variants in Smoking and Alcohol Use}

\author{
David M. Brazel, Yu Jiang, Jordan M. Hughey, Valérie Turcot, Xiaowei Zhan, Jian Gong, \\ Chiara Batini, J. Dylan Weissenkampen, MengZhen Liu, CHD Exome+ Consortium, Consortium \\ for Genetics of Smoking Behaviour, Daniel R. Barnes, Sarah Bertelsen, Yi-Ling Chou, \\ A. Mesut Erzurumluoglu, Jessica D. Faul, Jeff Haessler, Anke R. Hammerschlag, Chris Hsu, \\ Manav Kapoor, Dongbing Lai, Nhung Le, Christiaan A. de Leeuw, Anu Loukola, \\ Massimo Mangino, Carl A. Melbourne, Giorgio Pistis, Beenish Qaiser, Rebecca Rohde, \\ Yaming Shao, Heather Stringham, Leah Wetherill, Wei Zhao, Arpana Agrawal, Laura Bierut, \\ Chu Chen, Charles B. Eaton, Alison Goate, Christopher Haiman, Andrew Heath, \\ William G. lacono, Nicholas G. Martin, Tinca J. Polderman, Alex Reiner, John Rice, \\ David Schlessinger, H. Steven Scholte, Jennifer A. Smith, Jean-Claude Tardif, Hilary A. Tindle, \\ Andries R. van der Leij, Michael Boehnke, Jenny Chang-Claude, Francesco Cucca, \\ Sean P. David, Tatiana Foroud, Joanna M.M. Howson, Sharon L.R. Kardia, Charles Kooperberg, \\ Markku Laakso, Guillaume Lettre, Pamela Madden, Matt McGue, Kari North, \\ Danielle Posthuma, Timothy Spector, Daniel Stram, Martin D. Tobin, David R. Weir, \\ Jaakko Kaprio, Gonçalo R. Abecasis, Dajiang J. Liu, and Scott Vrieze
}

\begin{abstract}
BACKGROUND: Smoking and alcohol use have been associated with common genetic variants in multiple loci. Rare variants within these loci hold promise in the identification of biological mechanisms in substance use. Exome arrays and genotype imputation can now efficiently genotype rare nonsynonymous and loss of function variants. Such variants are expected to have deleterious functional consequences and to contribute to disease risk.

METHODS: We analyzed $\sim 250,000$ rare variants from 16 independent studies genotyped with exome arrays and augmented this dataset with imputed data from the UK Biobank. Associations were tested for five phenotypes: cigarettes per day, pack-years, smoking initiation, age of smoking initiation, and alcoholic drinks per week. We conducted stratified heritability analyses, single-variant tests, and gene-based burden tests of nonsynonymous/ loss-of-function coding variants. We performed a novel fine-mapping analysis to winnow the number of putative causal variants within associated loci.

RESULTS: Meta-analytic sample sizes ranged from 152,348 to 433,216, depending on the phenotype. Rare coding variation explained $1.1 \%$ to $2.2 \%$ of phenotypic variance, reflecting $11 \%$ to $18 \%$ of the total single nucleotide polymorphism heritability of these phenotypes. We identified 171 genome-wide associated loci across all phenotypes. Fine mapping identified putative causal variants with double base-pair resolution at 24 of these loci, and between three and 10 variants for 65 loci. Twenty loci contained rare coding variants in the $95 \%$ credible intervals.

CONCLUSIONS: Rare coding variation significantly contributes to the heritability of smoking and alcohol use. Finemapping genome-wide association study loci identifies specific variants contributing to the biological etiology of substance use behavior.
\end{abstract}

Keywords: Alcohol, Behavioral genetics, GWAS, Heritability, Nicotine, Tobacco

https://doi.org/10.1016/j.biopsych.2018.11.024 
Tobacco and alcohol use together account for more morbidity and mortality in Western society than any other single risk factor or health condition (1). These preventable and modifiable behaviors are heritable (2), but previous human and model organism research, including genome-wide association studies (GWASs) of common variants, have resulted in few associated genetic variants, which most prominently feature genes involved in alcohol/nicotine metabolism and nicotinic receptors (3-7).

Advances in sequencing, genotyping, and genotype imputation now allow cost-effective investigation of rare and low frequency variants. Compared with common variants (minor allele frequency [MAF] $>1 \%$ ) most commonly used in GWASs, rare variants have greater potential to elucidate biological mechanisms of complex traits, including substance use and addiction $(8,9)$. In particular, nonsynonymous and loss-offunction coding variants, which result in the loss of normal function of a protein, may have greater phenotypic impact and more direct mechanistic interpretation than other variants that do not have obvious biological consequences $(10,11)$.

No large-scale genome- or exome-wide study of rare variation has been conducted to date. The vast majority of existing addiction-related rare variant studies have used targeted sequencing of putative addiction-associated loci to discover and test for association in relatively small samples. Existing research has led to intriguing leads, including rare variant associations in loci that span nicotinic receptor gene clusters (12-21) and alcohol metabolism genes (22-24) for nicotine and alcohol dependence, respectively. This strategy has also produced rare variant associations in novel loci. In one case, gene-level association tests were used to find an association with rare variants in SERINC2 (24). In another case, a burden test across PTP4A1, PHF3, and EYS showed association with alcohol dependence (25). Unfortunately, these genes are not obviously involved in etiological processes related to addiction, and replications have not been reported to date.

Previous studies have also attempted to leverage information about predicted functional consequences of rare mutations to improve association analyses. One study of nicotine dependence found significant rare single-variant associations in CHRNB4, but only when variants were weighted by their predicted effect on the cellular response to nicotine and acetylcholine (26). Such positive findings could benefit from replication, which has not always been straightforward. For example, all rare variant associations in addiction are, to our knowledge, candidate gene analyses with type I error thresholds based only on the number of tests within that region. Historically, such analyses have produced overly optimistic estimates of the number of associated loci (27). Genome-wide analyses with more conservative type I error thresholds have reported null rare variant findings across an array of phenotypes relevant to addiction (28-30). Precisely because genome-wide analyses are conducted on many variants across the genome, they are in principle able to discover novel rare variant associations within new or known loci. One way to improve power in genome-wide analyses is through genetic association meta-analysis, which entails the aggregation of results across many studies to achieve large sample sizes.

Here, we attempted to expand on these previous discoveries by conducting the largest meta-analytic investigation of exonic rare variants to date. We conducted an exome-wide association meta-analysis of nicotine and alcohol use across 16 studies genotyped on the exome array, which genotypes low-frequency nonsynonymous and putative loss-of-function exonic variants. We combined these data with the UK Biobank, which includes approximately 400,000 individuals of European ancestry with genotype imputation to the Haplotype Reference Consortium (31) imputation reference panel and relevant smoking/drinking phenotypes. Sample sizes for wellimputed variants were thus enlarged, and the availability of noncoding variants from UK Biobank enabled comprehensive analysis of genetic architecture (32) and fine mapping (33).

We conducted single-variant and gene-based tests of association with five smoking and drinking phenotypes. We applied a novel fine-mapping analysis to prioritize causal variants using statistical and functional information. We also evaluated the contribution of rare exonic variants to the heritability of these phenotypes. Family studies, as well as studies of the aggregate effects of common variants, have found both alcohol use and tobacco use to be heritable behaviors (30,34-38). Research on the aggregate contribution of rare variants, however, has been scarce, with previous work on related phenotypes in smaller samples failing to detect aggregate effects for smoking and alcohol consumption (28). We used meta-analytic summary statistics to quantify the contribution to heritability of variants in various functional categories and frequency bins.

\section{METHODS AND MATERIALS}

Seventeen studies contributed summary statistics for metaanalysis. These studies, their sample sizes, and available phenotypes are listed in Tables S1 and S2 in Supplement 1. We augmented our 16 exome chip cohorts with the UK Biobank, in which imputation to the Haplotype Reference Consortium panel was used in lieu of an exome chip array. All individuals were of European ancestry, as determined by genetic principal components.

\section{Phenotypes}

Phenotypes were selected to represent multiple stages of smoking. These included initiation, heaviness of use among smokers, and a measure of total lifetime exposure to tobacco. For alcohol use only, a measure of amount of alcohol use was systematically available across studies. The selected phenotypes are relevant to prior GWASs of smoking and alcohol use; are commonly available in psychological, medical, and epidemiological datasets; and are known to be correlated with measures of substance dependence (4,39-41).

1. Cigarettes per day: the average number of cigarettes smoked in a day among current and former smokers. Studies with binned responses used their existing bins. Studies that recorded an integer value binned responses into one of four categories: $1=1$ to $10,2=11$ to $20,3=$ 21 to $30,4=31$ or more. Anyone reporting zero cigarettes per day was coded as missing. This phenotype is a component of commonly used measures of nicotine dependence such as the Fagerström Test for Nicotine Dependence. 
2. Pack-years: defined in the same way as cigarettes per day but not necessarily binned, divided by 20 (cigarettes in a pack), and multiplied by number of years smoking. This yielded a measure of total overall exposure to tobacco and is relevant to disease outcomes for which smoking is a risk factor, such as cancer and chronic obstructive pulmonary disease risk.

3. Age of initiation of smoking: a measure of early cigarette use. Defined as the age at which a participant first started smoking regularly.

4. Smoking initiation: a binary variable of whether the individual had ever been a regular smoker (1) or not (0), and often defined as having smoked at least 100 cigarettes during his or her lifetime.

5. Drinks per week: a measure of drinking frequency/quantity. The average number of drinks per week in current or former drinkers.

\section{Genotypes}

Fourteen of the 17 studies were genotyped with the Illumina HumanExome BeadChip (Illumina, San Diego, CA), which contains $\sim 250,000$ low-frequency nonsynonymous variants, variants from the GWAS catalog, and a small number of variants selected for other purposes. Two studies were genotyped on the Illumina Human Core Exome, which includes an additional $\sim 250,000$ tag single nucleotide polymorphisms (SNPs). The remaining study, the UK Biobank, was imputed using Haplotype Reference Consortium panel $(31,42)$, as well as the reference panel by UK $10 \mathrm{~K}$ and 1000 Genomes Project. An integrated callset was released by the UK Biobank team (42). Our UK Biobank genetic association analyses were conducted based on the integrated callset with additional quality control.

\section{Generation of Summary Association Statistics}

Seventeen independent studies (see Table S1 in Supplement 1) with smoking and drinking phenotypes were included in the discovery phase. Individual studies conducted association analysis accounting for age, sex, any study-specific covariates, and ancestry principal components (see Table S2 in Supplement 1 for genomic control values), and they submitted summary statistics for meta-analysis. For studies with related individuals (see Table S1 in Supplement 1), relatedness was accounted for in linear mixed models using empirically estimated kinships from common SNPs (43). Residuals were inverse-normalized to help ensure well-behaved test statistics for rare variant tests.

Quality control of per-study summary statistics included evaluation and correction of strand flips and allele flips through systematic comparison of alleles and allele frequencies against the reference datasets ExAC v2.0, 1000 Genomes Phase 3, and dbSNP. Variants with call rates $<0.9$ or Hardy-Weinberg $p<1 \times 10^{-7}$ were also removed. The latter filter was meant to avoid findings that could not be more broadly replicated across the 17 studies.

\section{Meta-analysis}

Association testing was done in stages. First, we conducted genome-wide association meta-analysis. Variants with $p$ values less than the genome-wide significance threshold of $5 \times 10^{-8}$ were deemed statistically significant. Loci were defined as 1 million base-pair windows surrounding a "sentinel" (most significant) variant in the locus. Overlapping or adjacent loci were combined into a single locus. Conditional analysis and fine mapping was then performed within each locus. We attempted to replicate one very rare variant (rs36015615 in STARD3 associated with cigarettes per day) (see Results and Table 1) that was available in two other exome chip consortia: the CHD Exome+ Consortium $(N=17,789)$ and the Consortium for Genetics of Smoking Behaviour $(N=$ 28,583). Both consortia defined their phenotypes, including cigarettes per day, similarly, as the usual number of cigarettes smoked in a day corrected for sex, age, and principal components (and/or genetic relatedness, as appropriate), and both consortia inverse-normalized the data before association analysis.

We also conducted gene-level association tests grouping nonsynonymous, stop-gain, stop-loss, and splice variants within each gene, using rareMETALS version 6.0 (44). Variant annotation was conducted using SEQMINER with RefSeq 1.9 (45). Two complementary gene-level association tests were performed: the sequence kernel association test $(46,47)$ with an MAF cutoff of $1 \%$, and a simple burden test (48) that summed the number of rare alleles within a given gene, again with a maximum MAF of $1 \%$. We chose variants with MAF $\leq 1 \%$ because we were interested in the contribution of variants with a frequency lower than that which has been reliably imputed and tested in past GWAS meta-analyses. We considered a gene association to be significant if the $p$ value surpassed a Bonferroni correction for the number of genes tested for a given phenotype and test, assuming approximately 20,000 genes in the genome $\left(.05 / 20,000=2.5 \times 10^{-6}\right)$.

We performed iterative conditional analysis using a partial correlation-based score (PCBS) statistic (49), which can perform proper conditional analysis for meta-analysis that combines datasets measured using different arrays. PCBS takes GWAS meta-analysis summary statistics and linkage disequilibrium (LD) estimated from the Haplotype Reference Consortium panel as input.

As a key step to evaluate the contribution of variants within a genome-wide significant locus (33), we used our PCBS framework to apply two complementary fine-mapping techniques to identify putatively causal genetic variants. The first technique was a Bayesian approach described previously (50) that estimates the posterior probability of association based on the statistical strength of the association for variants in each locus. We also applied a version of fgwas (51) modified to work within the PCBS, which assumes that variants in different functional categories have potentially different prior probability of association. For loci with a single association signal, effect sizes and variance from single-SNP analyses were used. If a locus contained multiple signals, we used effect sizes and variance from conditional analysis adjusting for all other index variants in this region.

Finally, we attempted to replicate previous rare variant associations referenced in the introduction and listed in Table S4 in Supplement 2. We attempted replication in our phenotypes for any single variant when that variant was directly genotyped or imputed. We applied a liberal threshold that corrected only 


\begin{tabular}{|c|c|c|c|c|c|c|c|c|c|c|c|c|}
\hline \multirow[b]{2}{*}{ SNP } & \multirow[b]{2}{*}{ REF/ALT } & \multirow[b]{2}{*}{$n^{a}$} & \multirow[b]{2}{*}{ ALT AF } & \multirow[b]{2}{*}{$\begin{array}{l}\text { GWAS } \\
p \text { Value }\end{array}$} & \multirow[b]{2}{*}{$B$} & \multirow[b]{2}{*}{ SE } & \multirow[b]{2}{*}{ Direction } & \multirow[b]{2}{*}{ Annotation } & \multicolumn{2}{|c|}{$\begin{array}{l}\text { Posterior Probability of } \\
\text { Association }\end{array}$} & \multicolumn{2}{|c|}{$\begin{array}{c}\text { Number SNPs (Low-Frequency } \\
\text { Coding SNPs) in 95\% Credible } \\
\text { Interval }\end{array}$} \\
\hline & & & & & & & & & $\begin{array}{c}\text { Without } \\
\text { Functional Prior }\end{array}$ & $\begin{array}{l}\text { With Functional } \\
\text { Prior (fgwas) }\end{array}$ & $\begin{array}{c}\text { Without } \\
\text { Functional Prior }\end{array}$ & $\begin{array}{c}\text { With Functional } \\
\text { Prior (fgwas) }\end{array}$ \\
\hline \multicolumn{13}{|c|}{ Cigarettes per Day } \\
\hline rs $36015615^{b}$ & $\mathrm{G} / \mathrm{A}$ & 69,951 & .0002 & $3.2 \times 10^{-8}$ & 1.2 & 0.210 & $==+=++=+=\mathrm{X}=\mathrm{X}+\mathrm{X}+++$ & $\begin{array}{l}\text { Nonsynonymous } \\
\text { [STARD3] }\end{array}$ & .82 & .62 & 8997 (6211) & $11,302(6232)$ \\
\hline rs16969968 & $\mathrm{G} / \mathrm{A}$ & 153,918 & .34 & $2.5 \times 10^{-139}$ & 0.096 & 0.0038 & +-++-+++++-++++++ & $\begin{array}{l}\text { Nonsynonymous } \\
\text { [CHRNA5] }\end{array}$ & .84 & .92 & $2(0)$ & $2(0)$ \\
\hline \multicolumn{13}{|c|}{ Drinks per Week } \\
\hline rs1260326 & $\mathrm{T} / \mathrm{C}$ & 357,854 & .61 & $4.6 \times 10^{-40}$ & 0.032 & 0.0024 & +++++++++--++++++ & $\begin{array}{l}\text { Nonsynonymous } \\
\text { [GCKR] }\end{array}$ & 1.0 & 1.0 & $1(0)$ & $1(0)$ \\
\hline rs1229984 & $\mathrm{T} / \mathrm{C}$ & 334,588 & .98 & $2.3 \times 10^{-173}$ & 0.25 & 0.0088 & $=+-\mathrm{XXXX}+\mathrm{XXXX}=++++$ & $\begin{array}{l}\text { Nonsynonymous } \\
{[A D H 1 B]}\end{array}$ & 1.0 & 1.0 & $1(1)$ & $1(1)$ \\
\hline rs28929474 & $\mathrm{C} / \mathrm{T}$ & 357,854 & .02 & $2.2 \times 10^{-11}$ & -0.057 & 0.0085 & --++------+++----+ & $\begin{array}{c}\text { Nonsynonymous } \\
\text { [SERPINA1] }\end{array}$ & .95 & 1.0 & $1(1)$ & $1(1)$ \\
\hline rs1800566 & $\mathrm{G} / \mathrm{A}$ & 357,854 & .18 & $2.00 \times 10^{-8}$ & 0.017 & 0.0031 & +++++++++++--+++- & $\begin{array}{l}\text { Nonsynonymous } \\
\text { [NQO1] }\end{array}$ & .32 & .97 & $103(0)$ & $1(0)$ \\
\hline \multicolumn{13}{|c|}{ Smoking Initiation } \\
\hline rs2232423 & $\mathrm{A} / \mathrm{G}$ & 433,216 & .11 & $1.40 \times 10^{-8}$ & -0.019 & 0.0034 & -+-+---+------ & $\begin{array}{l}\text { Nonsynonymous } \\
\text { [ZSCAN12] }\end{array}$ & .84 & .64 & $502(0)$ & $2(0)$ \\
\hline rs35891966 & $\mathrm{G} / \mathrm{A}$ & 433,216 & .07 & $1.30 \times 10^{-8}$ & -0.024 & 0.0042 & ---+-+---++--- & $\begin{array}{l}\text { Nonsynonymous } \\
\text { [NAV2] }\end{array}$ & .98 & 1.0 & $1(0)$ & $1(0)$ \\
\hline rs 147052174 & $\mathrm{G} / \mathrm{T}$ & 433,216 & .02 & $1.2 \times 10^{-7}$ & 0.043 & 0.0080 & +++++++++-++++ & $\begin{array}{l}\text { Nonsynonymous } \\
{[F A M 163 A]}\end{array}$ & .81 & 1.0 & $2432(66)$ & $1(0)$ \\
\hline rs6265 & $\mathrm{C} / \mathrm{T}$ & 433,216 & .19 & $1.9 \times 10^{-10}$ & -0.017 & 0.0030 & ++-++-++--+--- & $\begin{array}{l}\text { Nonsynonymous } \\
{[B D N F]}\end{array}$ & .32 & .83 & $25(0)$ & $2(0)$ \\
\hline rs61754158 & $\mathrm{C} / \mathrm{T}$ & 433,216 & .01 & $1.4 \times 10^{-6}$ & -0.055 & 0.0114 & $--+-+---=+=-+-$ & $\begin{array}{c}\text { Nonsynonymous } \\
{[\text { HEATR5A] }}\end{array}$ & .39 & .87 & $9742(195)$ & $9742(195)$ \\
\hline rs34967813 & $\mathrm{A} / \mathrm{G}$ & 433,216 & .31 & $8.1 \times 10^{-7}$ & -0.011 & 0.0023 & -----+--+--+--- & $\begin{array}{l}\text { Nonsynonymous } \\
\text { [RYR2] }\end{array}$ & .14 & .98 & $7413(56)$ & $1(0)$ \\
\hline
\end{tabular}

A variant is considered "rare" if minor allele frequency (MAF) $<0.01$, and low frequency if $0.01<$ MAF $<0.05$. In the Direction column each symbol represents the contribution of one of the studies. A plus sign $(+)$ indicates that the ALT allele had a positive effect in that study, a minus $(-)$ indicates a negative effect, an equals sign $(=)$ indicates that the variant was monomorphic, and an $\mathrm{X}$ indicates that it was absent in that study. The order of studies for cigarettes per day and drinks per week was ARIC, UKB, COGA, FINNTWIN, FUSION, GECCO, HRS, ID1000, MEC, METSIM, MHI, MCTFR, NAGOZALC, NESCOG, SardiNIA, TwinsUK, and WHI. For smoking initiation, the order is the same, except COGA and MCTFR were not available. See Table S1 in Supplement 1 for study acronym explanations.

ALT, alternate allele; ALT AF, allele frequency of the alternate allele estimated in the meta-analysis; GWAS, genome-wide association study; REF, reference allele on GRCh37; SNP, single nucleotide polymorphism.

${ }^{a}$ Across all studies that genotyped the variant.

${ }^{b}$ rs36015615 did not replicate in two additional datasets. See Results section. 
for the number of tests conducted for this replication exercise $(.05 / 46=.001)$.

\section{Genetic Architecture}

We performed heritability and genetic correlation analyses using LD score regression (52). The method calculates LD scores from the Haplotype Reference Consortium, and the estimation of heritability with these LD scores then follows established methods (53,54). Heritability was estimated for each trait and partitioned by annotation category and frequency bins. First, we annotated variants on the exome chip based on gene definitions in RefSeq 1.9, using SEQMINER version 6.0 (55). A variant is classified as coding if it belongs to either one of the following categories: nonsynonymous, stop gain, stop loss, and splice. Seven functional categories were considered in the model, including intergenic, intron, common coding (MAF $>0.01$ ), rare coding (MAF $<0.01$ ), synonymous, and $3^{\prime} / 5^{\prime}$ untranslated regions. We fitted the baseline model with seven categories and estimated phenotypic variance explained by each category.

\section{RESULTS}

GWAS analyses behaved well, with genomic control values for the GWAS across exome chip and UK Biobank imputed variants between 1.05 and 1.3. The intercept for LD score regression ranged between 0.99 and 1.1, indicating absent or minimal effects of population stratification (per-study genomic control values can be found in Table S2 in Supplement 1). A total of 171 loci were identified under the genome-wide significance threshold $\left(p<5 \times 10^{-8}\right)$, including $3,11,17,93$, and 47 loci for age of initiation of smoking, cigarettes per day, pack-years, smoking initiation, and drinks per week, respectively. A list of all sentinel variants within each locus is shown in Table S5 in Supplement 2. QQ plots and Manhattan plots are available in Figures $\mathrm{S} 1$ and $\mathrm{S} 2$ in Supplement 1 (additional exploratory GWAS meta-analyses of individuals with significant African ancestry are provided in Supplement 1 [including up to 8974 individuals from three studies]) (see also Table S3 and Figures S3 and S4 in Supplement 1). The genome-wide significant association results included known loci associated with smoking and alcohol use phenotypes. These included associations between smoking phenotypes and variants within the CHRNA5-CHRNA3-CHRNB4 nicotinic receptor cluster, nicotine metabolism gene CYP2A6, and a locus near dopamine receptor $D R D 2$. We also replicated previous associations between nonsynonymous variant rs1229984 in ADH1B and drinks per week. Only one very rare variant was associated with any of our five phenotypes. This was rs36015615 (MAF = 0.0002 ), a nonsynonymous variant in STARD3, associated with cigarettes per day $\left(p=3.2 \times 10^{-8}\right)$. This novel variant did not replicate in either of two replication consortium datasets, the CHD Exome+ Consortium ( $N=17,789 ; B=-0.01, p=.94)$ or the Consortium for Genetics of Smoking Behaviour $(N=$ 28,583; $B=0.056, p=.84)$. Based on the estimated genetic effects in the discovery sample $(\beta=1.2)$, the power for replication is $>99 \%$. However, if we assume that the observed effect sizes in the replication datasets are correct, there is $5 \%$ power for replication based on this estimated effect. The pattern of results may be due to winner's curse, or the discovered variant may be a false positive finding. Additional studies are required to narrow the possible interpretations.

The fine-mapping analysis of all 171 GWAS loci pinpointed putatively causal variants with high resolution in some cases. The $95 \%$ credible interval for $34 \%$ of the loci had $<10$ SNPs and 24 loci had double base-pair resolution, including several instances in which the sole putative causal variant was nonsynonymous and of lower frequency, although in only one case with $\mathrm{MAF}<1 \%$. The resolution increased somewhat when functional information was used to inform the prior probability, with double base-pair resolution at 32 loci, and $44 \%$ of loci having $<10$ SNPs in the $95 \%$ credible interval. Table 1 includes all nonsynonymous or loss-of-function variants within the genome-wide significant loci that had a posterior probability of association $>.80$ from at least one of the fine-mapping methods. Additional results from the fine-mapping analysis are available in Tables S6 and S7 in Supplement 2. Several known functional variants were identified through this method, including rs16969968 (56), a nonsynonymous variant in nicotinic receptor gene CHRNA5 associated with cigarettes per day (posterior probability of association [PPA] $=.92$ and .84 from the fine-mapping analysis with and without functional priors, respectively); rs1229984 (57), a nonsynonymous variant in alcohol metabolism gene $A D H 1 B$ associated with drinks per week (PPA = 1.0 and 1.0); and, although with somewhat weaker evidence, rs6265 (58), a nonsynonymous variant in brain-derived neurotrophic factor $B D N F$ associated with smoking initiation (MAF $=0.19 ;$ PPA $=.83$ and .32 ).

Novel variants in novel genes were also prioritized at high resolution. To take the most statistically compelling examples in Table 1, we found that rs28929474, a low-frequency nonsynonymous variant in SERPINA1, was associated with drinks per week (MAF = 0.02; PPA = 1.0 and .95). When homozygous, the alternate $T$ (allele frequency $=0.02$; frequency of $T \mathrm{~T}$ genotype under Hardy-Weinberg $=4$ in 10,000) allele is a leading cause of $\alpha_{1}$-antitrypsin deficiency. Here, we find that the same risk allele, the $T$ allele, is associated with approximately a $0.05-$ SD decrease in drinks per week. We also discovered that rs35891966, a variant in NAV2, was associated with smoking initiation (MAF $=0.07$; PPA $=1.0$ and .98 ) at single base-pair resolution. NAV2 is involved in neuronal development and was previously shown to be differentially expressed between smokers and nonsmokers, but not previously implicated in GWASs (59).

Results of gene-based tests are provided in Table 2. A novel gene, rho guanine nucleotide exchange factor 37 (ARHGEF37), was associated with age of initiation of smoking $\left(p=1.9 \times 10^{-6}\right)$. ARHGEF37 has not been widely studied and its function in not well known. Another novel gene without an immediate biological interpretation, was HEAT repeat-containing protein 5A (HEATR5A), associated with smoking initiation $\left(p=1.4 \times 10^{-8}\right)$. We also discovered a significant gene-based association between known alcohol metabolism gene $A D H 1 C$ and drinks per week $(p=$ $1.4 \times 10^{-27}$ and $p=1.9 \times 10^{-40}$ from the burden and sequence kernel association test tests, respectively). Finally, even with relaxed $p$ value thresholds, we failed to replicate genes identified in previous rare variant association studies referenced in the introduction (Table S4 in Supplement 2), with the exception of ADH1C and CHRNA5, two loci long known to be associated with alcohol use and smoking, respectively. 
Table 2. Significant Gene-Based Test Results, Assuming a Bonferroni Threshold of .05/20,000 $=2.5 \times 10^{-6}$

\begin{tabular}{llcccccc}
\hline Phenotype & Gene & $n$ & Variants & $B$ & SE & $p$ Value & Method \\
\hline Age of Initiation of Smoking & ARHGEF37 & 147,010 & 17 & 0.08 & 0.017 & $1.9 \times 10^{-6}$ & Burden \\
\hline Smoking Initiation & HEATR5A & 427,262 & 41 & -0.02 & 0.009 & $1.4 \times 10^{-8}$ & SKAT \\
\hline Drinks per Week & ADH1C & 353,265 & 4 & -0.15 & 0.014 & $1.8 \times 10^{-27}$ & Burden \\
\hline Drinks per Week & ADH1C & 353,265 & 4 & -0.15 & 0.014 & $1.9 \times 10^{-40}$ \\
\hline
\end{tabular}

No significant genes were identified for the other two phenotypes.

SKAT, sequence kernel association test.

The estimated total SNP heritability for age of initiation of smoking, cigarettes per day, pack-years, smoking initiation, and drinks per week was $6 \%, 9 \%, 10 \%, 14 \%$, and $16 \%$, respectively. Significant phenotypic variance was explained by rare nonsynonymous variants for all traits, ranging from $1.0 \%$ to $2.2 \%$ (Table 3 ). As a fraction of the SNP heritability, rare nonsynonymous variants accounted for $11 \%$ to $18 \%$. Results for all seven functional categories are listed in Table S8 in Supplement 2; appreciable heritability was accounted for by common and rare coding variants, and intergenic variants. Variants in the untranslated regions and intronic regions contributed less. Almost all pairs of phenotypes were genetically correlated (Table 4), and the directions of the genetic correlations were in the expected direction. For instance, cigarettes per day was positively correlated with drinks per week $(0.2 \pm$ 0.09), consistent with the observation that increased alcohol consumption is correlated with increased tobacco consumption. Age of initiation of smoking has a negative correlation with all other traits, which is consistent with the observation that an earlier age of smoking initiation is correlated with increased tobacco and alcohol consumption in adulthood. The patterns and magnitudes of correlation are highly similar when considering only rare nonsynonymous variants (Table 4).

Table 3. Estimation of Heritability Explained by Variants on Exome Array

\begin{tabular}{|c|c|c|c|c|}
\hline \multirow[b]{2}{*}{ Annotation } & \multirow[b]{2}{*}{ Phenotype } & \multicolumn{3}{|c|}{ Heritability Estimates } \\
\hline & & $\overline{\hat{h}^{2}}$ & $\operatorname{se}\left(\widehat{h}^{2}\right)$ & $p$ Value \\
\hline \multirow[t]{5}{*}{ All Variants } & $\begin{array}{l}\text { Age of initiation } \\
\text { of smoking }\end{array}$ & .06 & .0049 & $7.7 \times 10^{-35}$ \\
\hline & Cigarettes per day & .09 & .0019 & $<2.2 \times 10^{-303}$ \\
\hline & Pack-years & .10 & .0022 & $<2.2 \times 10^{-303}$ \\
\hline & Smoking initiation & .14 & .0007 & $<2.2 \times 10^{-303}$ \\
\hline & Drinks per week & .16 & .0089 & $7.3 \times 10^{-73}$ \\
\hline \multirow{5}{*}{$\begin{array}{l}\text { Rare Coding } \\
\text { Variants } \\
\text { (MAF }<0.01)\end{array}$} & $\begin{array}{l}\text { Age of initiation } \\
\text { of smoking }\end{array}$ & .011 & .0015 & $2.8 \times 10^{-2}$ \\
\hline & Cigarettes per day & .010 & .0006 & $1.7 \times 10^{-2}$ \\
\hline & Pack-years & .018 & .0007 & $8.5 \times 10^{-6}$ \\
\hline & Smoking initiation & .022 & .0002 & $3.9 \times 10^{-16}$ \\
\hline & Drinks per week & .020 & .0013 & $1.8 \times 10^{-7}$ \\
\hline
\end{tabular}

We estimate the heritability based on a baseline model with seven different functional categories. The reported heritability $\widehat{h}^{2}$ is based on the cumulative value from the functional categories with significant heritabilities. We also report the $\mathrm{SD}\left(\operatorname{se}\left(\widehat{h}^{2}\right)\right)$ and $p$ values, estimated using jackknife resampling.

MAF, minor allele frequency.

\section{DISCUSSION}

With a maximum sample size ranging from 152,348 to 433,216 , the present study is the largest study to date of lowfrequency nonsynonymous and loss-of-function variants in smoking and alcohol use. Our meta-analytic study design combined studies genotyped on the exome array with imputed genotypes in the UK Biobank and allowed us to comprehensively evaluate the contribution of rare and low-frequency variants to the etiology of tobacco and alcohol use. All told, we identified 171 genome-wide significant loci for the five phenotypes.

We showed that the rare variants (MAF $\leq 1 \%$ ) together explain $1.0 \%$ to $2.2 \%$ of the phenotypic variance for the five traits, amounting to $11 \%$ to $18 \%$ of the total SNP heritability. A number of putatively causal low-frequency nonsynonymous variants in novel genes were identified through two complementary fine-mapping techniques. These include a variant known to affect $\alpha_{1}$-antitrypsin deficiency in SERPINA1. The effect of the risk allele resulted in a decrease in drinks per week. One interpretation is that this variant leads to impaired liver function through $\alpha_{1}$-antitrypsin deficiency, which, in turn, reduces alcohol consumption. Interestingly, neither this particular variant nor the locus surrounding it was associated with smoking phenotypes, even though $\alpha_{1}$-antitrypsin deficiency also affects lung function over time. Other mechanisms by which SERPINA1 exerts its effect on alcohol consumption are certainly possible. Another novel nonsynonymous variant was in neuron navigator 2 (NAV2), associated with smoking initiation. NAV2 has not previously been associated with substance use or addiction. Given its suspected involvement in neuronal growth and migration, a putatively causal nonsynonymous variant is a strong candidate for functional follow up experiments. Other genes implicated in the fine-mapping analysis have less direct interpretations (e.g., HEATR5A), and such results will benefit from replication and/or follow-up experiments. In general, fine-mapping studies narrowed the credible set of likely causal variants to single or double basepair resolution for 24 loci (Table S6 in Supplement 2). Some loci were not amenable to fine mapping, with credible intervals containing thousands of SNPs in some cases. Given the high cost in money and time of conducting functional experiments at the cellular or organismal level, fine-mapping likely causal variants can be extremely useful in predicting functional consequences and prioritizing variants for further work.

Gene-based tests identified a small number of associated genes, including an expected association with $A D H 1 C$ and drinks per week. The other two associated genes, ARHGEF37 and HEATR5A, do not lend themselves to ready biological interpretations. 
Table 4. Estimation of Genetic Correlation Between Smoking and Drinking Traits

\begin{tabular}{|c|c|c|c|c|}
\hline \multirow[b]{2}{*}{ Trait 1} & \multirow[b]{2}{*}{ Trait 2} & \multicolumn{3}{|c|}{ Genetic Correlation } \\
\hline & & $\widehat{r}_{g}$ & $\operatorname{se}\left(\widehat{r}_{g}\right)$ & $p$ Value \\
\hline \multicolumn{5}{|c|}{ Aggregated Genetic Correlation Induced by All Variants on the Exome Array } \\
\hline Drinks per Week & Smoking initiation & 0.43 & 0.06 & $1.7 \times 10^{-11}$ \\
\hline Drinks per Week & Age of initiation of smoking & 0.01 & 0.13 & $9.3 \times 10^{-1}$ \\
\hline Drinks per Week & Pack-years & 0.22 & 0.10 & $2.6 \times 10^{-2}$ \\
\hline Drinks per Week & Cigarettes per day & 0.20 & 0.09 & $3.1 \times 10^{-2}$ \\
\hline Smoking Initiation & Age of initiation of smoking & -0.64 & 0.11 & $1.1 \times 10^{-8}$ \\
\hline Smoking Initiation & Pack-years & 0.45 & 0.08 & $4.9 \times 10^{-8}$ \\
\hline Smoking Initiation & Cigarettes per day & 0.10 & 0.07 & $1.5 \times 10^{-1}$ \\
\hline Age of Initiation of Smoking & Pack-years & -0.63 & 0.17 & $2.1 \times 10^{-4}$ \\
\hline Age of Initiation of Smoking & Cigarettes per day & -0.26 & 0.16 & $9.9 \times 10^{-2}$ \\
\hline Pack-years & Cigarettes per day & 0.77 & 0.13 & $2.2 \times 10^{-9}$ \\
\hline \multicolumn{5}{|c|}{ Genetic Correlation Induced by Rare (MAF $<1 \%$ ) Nonsynonymous Variants } \\
\hline Drinks per Week & Smoking initiation & 0.49 & 0.08 & $1.2 \times 10^{-10}$ \\
\hline Drinks per Week & Age of initiation of smoking & -0.04 & 0.30 & $8.9 \times 10^{-1}$ \\
\hline Drinks per Week & Pack-years & 0.08 & 0.02 & $2.7 \times 10^{-4}$ \\
\hline Drinks per Week & Cigarettes per day & 0.09 & 0.02 & $5.2 \times 10^{-5}$ \\
\hline Smoking Initiation & Age of initiation of smoking & -1.10 & 0.21 & $1.3 \times 10^{-7}$ \\
\hline Smoking Initiation & Pack-years & 0.63 & 0.08 & $1.5 \times 10^{-14}$ \\
\hline Smoking Initiation & Cigarettes per day & 0.23 & 0.08 & $3.3 \times 10^{-3}$ \\
\hline Age of Initiation of Smoking & Pack-years & -1.10 & 0.33 & $1.5 \times 10^{-3}$ \\
\hline Age of Initiation of Smoking & Cigarettes per day & -0.69 & 0.32 & $3.2 \times 10^{-2}$ \\
\hline Pack-years & Cigarettes per day & 0.87 & 0.14 & $1.4 \times 10^{-9}$ \\
\hline
\end{tabular}

We estimate genetic correlations between five smoking and drinking traits. For genetic correlation estimates $\left(\widehat{r}_{g}\right)$, their SD $\left(\right.$ se $\left.\left(\widehat{r}_{g}\right)\right)$ and $p$ values are reported.

MAF, minor allele frequency.

We showed that rare coding variants available on the exome chip or imputable by the Haplotype Reference Consortium, with frequency $<1 \%$, explain significant proportions of phenotypic variance and a substantial proportion of the total SNP heritability. The exome chip was designed to genotype coding variants uncovered in $\sim 12,000$ sequenced exomes. By design, the exome chip comprehensively ascertained highconfidence rare nonsynonymous, splice, and stop variants within those sequences, and it only sparsely genotypes in other classes of variation, including common variants. The Haplotype Reference Consortium panel imputed data also have limited accuracy when the underlying genetic variants are rare. Therefore, our current investigation did not fully explore the genetic architecture of very rare variants (i.e., with MAF $<0.1 \%$ ). With the development of larger imputation reference panels, and the availability of large-scale deep whole-genome sequences (e.g., the TOPMed [Trans-Omics for Precision Medicine Study] study), we expect to be able to conduct an even more comprehensive analysis of the genetic architecture for variants with ever lower frequencies. Ultimately, the discovery of low frequency variants with small effects will require even larger sample sizes. For example, for rare variants with MAF of $0.1 \%$ and effects of $0.2,0.15$, and 0.1 SDs on the phenotype, to identify associations at $\alpha=5 \times 10^{-8}$ with $80 \%$ of power, sample sizes of $500,000,890,000$, and 1,990,000 are required. While such numbers seemed astronomical just a few years ago, they will indeed be attainable in the next few years with the availability of large biobank datasets and ever-improving imputation. Another limitation of the present study is the limited samples sizes from non-European ancestries, in which only exploratory analyses were possible. Substantial improvements can be made to the resolution of finemapping analysis by leveraging disparate LD information across samples with diverse ancestry (33). Future researchers will do well to include individuals of diverse ancestry.

\section{ACKNOWLEDGMENTS AND DISCLOSURES}

This research has been conducted using the UK Biobank Resource under Application Number 16651. This work was supported by the National Institute on Drug Abuse and the National Human Genome Research Institute of the National Institutes of Health Grant Nos. R01DA037904 (to SIV), R21DA040177 (to DJL), R01HG008983 (to DJL), R01GM126479 (to DJL), and 5T32DA01763713 (to DMB); funding sources listed in the Supplementary Note; and a National Science Foundation Graduate Research Fellowship (to JMH).

This material is based on work supported by the National Science Foundation Graduate Research Fellowship Program under Grant No. DGE1255832 (to JMH). Any opinions, findings, and conclusions or recommendations expressed in this material are those of the author(s) and do not necessarily reflect the views of the National Science Foundation.

The complete funding statements and acknowledgments for participating consortia are available in Supplement 1.

GRA is an employee of Regeneron Pharmaceuticals. All other individually named authors report no biomedical financial interests or potential conflicts of interest.

The members of the Consortium for Genetics of Smoking Behaviour report the following: Paul W. Franks has been a paid consultant for Eli Lilly and Sanofi Aventis and has received research support from several pharmaceutical companies as part of European Union Innovative Medicines 
Initiative (IMI) projects. Neil Poulter has received financial support from several pharmaceutical companies that manufacture either blood pressure lowering or lipid lowering agents or both and consultancy fees. Peter Sever has received research awards from Pfizer. Mark J. Caulfield is Chief Scientist for Genomics England, a UK government company.

\section{ARTICLE INFORMATION}

From the Institute for Behavioral Genetics (DMB) and Department of Molecular, Cellular, and Developmental Biology (DMB), University of Colorado Boulder, Boulder, Colorado; Department of Public Health Sciences (YJ, JMH, JDW) and Institute of Personalized Medicine (DJL), Penn State College of Medicine, Hershey, Pennsylvania; Department of Medicine (VT, J-CT, GL), Faculty of Medicine, Université de Montréal; and Montreal Heart Institute (VT, J-CT, GL), Montreal, Quebec, Canada; Department of Clinical Science (XZ), Center for Genetics of Host Defense, University of Texas Southwestern, Dallas, Texas; Public Health Sciences Division (JG, JH, CC, CK, AR), Fred Hutchinson Cancer Research Center; and Department of Epidemiology (CC, AR); and Department of Otolaryngology (CC), Head and Neck Surgery Center, University of Washington, Seattle, Washington; Department of Health Sciences (CB, AME, CAM, MDT), University of Leicester, Leicester; Department of Public Health and Primary Care (DRB, JMMH), University of Cambridge, Cambridge; and Department of Twin Research and Genetic Epidemiology (MM, TS), King's College London; and National Institute for Health Research Biomedical Research Centre at Guy's and St Thomas' Foundation Trust (MM), London, United Kingdom; Department of Psychology (ML, WGI, MM, SV), University of Minnesota, Minneapolis, Minnesota; Department of Neuroscience (SB, MK, AG), Icahn School of Medicine at Mount Sinai, New York; and Regeneron Pharmaceuticals (GRA), Tarrytown, New York; Survey Research Center (JDF, DRW), Institute for Social Research; and Department of Epidemiology (WZ, JAS, SLRK) and Department of Biostatistics (HS, MB, GRA), School of Public Health, University of Michigan, Ann Arbor, Michigan; Department of Complex Trait Genetics (ARH, CAdL, TJP, DP), Center for Neurogenomics and Cognitive Research, Amsterdam Neuroscience, VU University Amsterdam; Department of Clinical Genetics (DP), VU University Medical Centre; and Department of Psychology (HSS, ARvdL) and Amsterdam Brain and Cognition (HSS, ARvdL), University of Amsterdam, Amsterdam, the Netherlands; Department of Preventive Medicine (CHs, CHa, DS), Keck School of Medicine, University of Southern California, Los Angeles; and Department of Medicine (SPD), Stanford University, Stanford, California; Department of Medical and Molecular Genetics (DL, LW, TF), Indiana University School of Medicine, Indianapolis, Indiana; Department of Medical Microbiology (NL), Immunology and Cell Biology, Southern Illinois University School of Medicine, Springfield, Illinois; Institute for Molecular Medicine Finland (AL, BQ, JK) and Department of Public Health (AL, BQ, JK), University of Helsinki, Helsinki; and Department of Internal Medicine (ML), Institute of Clinical Medicine, University of Eastern Finland, Kuopio, Finland; Istituto di Ricerca Genetica e Biomedica (GP, FC), Consiglio Nazionale delle Ricerche, Monserrato, Italy; Department of Epidemiology (RR, YS, KN), University of North Carolina at Chapel Hill, Chapel Hill, North Carolina; Department of Psychiatry (Y-LC, AA, LB, AH, JR, PM), Washington University School of Medicine; and Department of Mathematics (JR), Washington University in St. Louis, St. Louis, Missouri; Department of Family Medicine (CBE), Brown University, Providence, Rhode Island; Queensland Institute for Medical Research (NGM), Brisbane, Australia; National Institute on Aging (DS), National Institutes of Health, Bethesda, Maryland; Department of Medicine (HAT), Vanderbilt University, Nashville, Tennessee; and the Division of Cancer Epidemiology (JC-C), German Cancer Research Center, Heidelberg, Germany.

CHD Exome+ Consortium: Praveen Surendran, Robin Young, Daniel R. Barnes, Sune Fallgaard Nielsen, Asif Rasheed, Maria Samuel, Wei Zhao, Jukka Kontto, Markus Perola, Muriel Caslake, Anton J.M. de Craen, Stella Trompet, Maria Uria-Nickelsen, Anders Malarstig, Dermot F. Reily, Maarten Hoek, Thomas Vogt, J. Wouter Jukema, Naveed Sattar, lan Ford, Chris J. Packard, Dewan S. Alam, Abdulla al Shafi Majumder, Emanuele Di Angelantonio, Rajiv Chowdhury, Philippe Amouyel, Dominique Arveiler, Stefan Blankenberg, Jean Ferrières, Frank Kee, Kari Kuulasmaa, Martina MüllerNurasyid, Giovanni Veronesi, Jarmo Virtamo, EPIC-CVD Consortium, Philippe Frossard, Børge Grønne Nordestgaard, Danish Saleheen, John Danesh, Adam S. Butterworth, Joanna M.M. Howson.
Consortium for Genetics of Smoking Behaviour: A. Mesut Erzurumluoglu, Victoria E. Jackson, Carl A. Melbourne, Tibor V. Varga, Helen R. Warren, Vinicius Tragante, loanna Tachmazidou, Sarah E. Harris, Evangelos Evangelou, Jonathan Marten, Weihua Zhang, Elisabeth Altmaier, Jian'an Luan, Claudia Langenberg, Robert A. Scott, Hanieh Yaghootkar, Kathleen Stirrups, Stavroula Kanoni, Eirini Marouli, Fredrik Karpe, Anna F. Dominiczak, Peter Sever, Neil Poulter, Olov Rolandsson, Clemens Baumbach, Saima Afaq, John C. Chambers, Jaspal S. Kooner, Nicholas J. Wareham, Frida Renström, Göran Hallmans, Riccardo E. Marioni, Janie Corley, John M. Starr, Niek Verweij, Rudolf A. de Boer, Peter van der Meer, Ersin Yavas, Ilonca Vaartjes, Michiel L. Bots, Folkert W. Asselbergs, Hans J. Grabe, Henry Völzke, Matthias Nauck, Stefan Weiss, Paul D.P. Pharoah, Alison M. Dunning, Joe G. Dennis, Deborah J. Thompson, Kyriaki Michailidou, Douglas F. Easton, Antonis C. Antoniou, Jessica Tyrrell, Evelin Mihailov, Nilesh J. Samani, Kaixin Zhou, Matthew J. Neville, Andres Metspalu, Colin N. A. Palmer, lan P. Hall, David P. Strachan, lan J. Deary, Tim M. Frayling, Caroline Hayward, Pim van der Harst, Eleftheria Zeggini, Understanding Society Scientific Group, Patricia B. Munroe, Jan-Håkan Jansson, Paul W. Franks, Panos Deloukas, Mark J Caulfield, Louise V. Wain, Martin D. Tobin.

$\mathrm{DMB}, \mathrm{YJ}$, and $\mathrm{JMH}$ contributed equally to this work.

This work was jointly supervised by SIV and DJL.

Address correspondence to Scott Vrieze, Ph.D., University of Minnesota, 75 East River Road, Minneapolis, MN 55455; E-mail: vrieze@umn.edu; or Dajiang J. Liu, Ph.D., Penn State College of Medicine, HCAR 2020, Hershey, PA 17033; E-mail: dajiang.liu@psu.edu.

Received Sep 15, 2017; revised Nov 5, 2018; accepted Nov 29, 2018.

Supplementary material cited in this article is available online at https:// doi.org/10.1016/j.biopsych.2018.11.024.

\section{REFERENCES}

1. Ezzati M, Lopez AD, Rodgers A, Vander Hoorn S, Murray CJL, Coll CRA (2002): Selected major risk factors and global and regional burden of disease. Lancet 360:1347-1360.

2. Polderman TJ, Benyamin B, de Leeuw CA, Sullivan PF, van Bochoven A, Visscher PM, et al. (2015): Meta-analysis of the heritability of human traits based on fifty years of twin studies. Nat Genet 47:702-709.

3. Eng MY, Luczak SE, Wall TL (2007): ALDH2, ADH1B, and ADH1C genotypes in Asians: A literature review. Alcohol Res Health 30:22-27.

4. Furberg $\mathrm{H}, \mathrm{Kim} \mathrm{Y}$, Dackor J, Boerwinkle E, Franceschini N Ardissino D, et al. (2010): Genome-wide meta-analyses identify multiple loci associated with smoking behavior. Nature Genet 42:441-447.

5. Saccone NL, Culverhouse RC, Schwantes-An TH, Cannon DS, Chen X, Cichon S, et al. (2010): Multiple independent loci at chromosome 15q25.1 affect smoking quantity: A meta-analysis and comparison with lung cancer and COPD. PLoS Genet 6:e1001053.

6. Bierut LJ, Stitzel JA (2014): Genetic contributions of the alpha 5 nicotinic receptor subunit to smoking behavior. Recept Ser 26:327-339.

7. Luczak SE, Glatt SJ, Wall TL (2006): Meta-analyses of ALDH2 and ADH1B with alcohol dependence in Asians. Psychol Bull 132 $607-621$.

8. Lek M, Karczewski KJ, Minikel EV, Samocha KE, Banks E, Fennell T, et al. (2016): Analysis of protein-coding genetic variation in 60,706 humans. Nature 536:285-291.

9. Minikel E, Lek M, Samocha KE, Karczewski KJ, Marshall JL, Armean I, et al . (2016): An early glimpse of saturation mutagenesis in humans: Insights from protein-coding genetic variation in 60,706 people. Prion 10:S107.

10. Sveinbjornsson G, Albrechtsen A, Zink F, Gudjonsson SA, Oddson A, Masson G, et al. (2016): Weighting sequence variants based on their annotation increases power of whole-genome association studies. Nat Genet 48:314-317.

11. Marouli E, Graff M, Medina-Gomez C, Lo KS, Wood AR, Kjaer TR, et al. (2017): Rare and low-frequency coding variants alter human adult height. Nature 542:186-190.

12. Yang J, Wang S, Yang Z, Hodgkinson CA, larikova P, Ma JZ, et al. (2014): The contribution of rare and common variants in 30 genes to risk nicotine dependence. Mol Psychiatry 20:1467-1478.

13. McClure-Begley TD, Papke RL, Stone KL, Stokes C, Levy AD, Gelernter J, et al. (2014): Rare human nicotinic acetylcholine receptor 
alpha4 subunit (CHRNA4) variants affect expression and function of high-affinity nicotinic acetylcholine receptors. J Pharmacol Exp Ther 348:410-420.

14. Piliguian M, Zhu AZ, Zhou Q, Benowitz NL, Ahluwalia JS, Sanderson Cox L, et al. (2014): Novel CYP2A6 variants identified in African Americans are associated with slow nicotine metabolism in vitro and in vivo. Pharmacogenet Genomics 24:118-128.

15. Haller G, Druley T, Vallania FL, Mitra RD, Li P, Akk G, et al. (2012): Rare missense variants in CHRNB4 are associated with reduced risk of nicotine dependence. Hum Mol Genet 21:647-655.

16. Haller G, Kapoor M, Budde J, Xuei X, Edenberg H, Nurnberger J, et al. (2014): Rare missense variants in CHRNB3 and CHRNA3 are associated with risk of alcohol and cocaine dependence. Hum Mol Genet 23:810-819.

17. Zuo L, Tan Y, Li C-SR, Wang Z, Wang K, Zhang X, et al. (2016): Associations of rare nicotinic cholinergic receptor gene variants to nicotine and alcohol dependence. Am J Med Genet Part B Neuropsychiatr Genet 171:1057-1071.

18. Xie P, Kranzler HR, Krauthammer M, Cosgrove KP, Oslin D, Anton RF, et al. (2011): Rare nonsynonymous variants in alpha-4 nicotinic acetylcholine receptor gene protect against nicotine dependence. Biol Psychiatry 70:528-536.

19. Wessel J, McDonald SM, Hinds Da, Stokowski RP, Javitz HS, Kennemer M, et al. (2010): Resequencing of nicotinic acetylcholine receptor genes and association of common and rare variants with the Fagerström Test for Nicotine Dependence. Neuropsychopharmacology 35:2392-2402.

20. Thorgeirsson TE, Steinberg S, Reginsson GW, Bjornsdottir G, Rafnar T, Jonsdottir I, et al. (2016): A rare missense mutation in CHRNA4 associates with smoking behavior and its consequences. Mol Psychiatry 21:594-600.

21. Olfson E, Saccone NL, Johnson EO, Chen L-S, Culverhouse R, Doheny K, et al. (2016): Rare, low frequency and common coding variants in CHRNA5 and their contribution to nicotine dependence in European and African Americans. Mol Psychiatry 21:601-607.

22. Peng Q, Gizer IR, Libiger O, Bizon C, Wilhelmsen KC, Schork NJ, et al. (2014): Association and ancestry analysis of sequence variants in ADH and ALDH using alcohol-related phenotypes in a Native American community sample. Am J Med Genet B Neuropsychiatr Genet 165B:673-683.

23. Way M, McQuillin A, Saini J, Ruparelia K, Lydall GJ, Guerrini I, et al. (2015): Genetic variants in or near ADH1B and ADH1C affect susceptibility to alcohol dependence in a British and Irish population. Addict Biol 20:594-604.

24. Zuo L, Wang K-S, Zhang X-Y, Li C-sR, Zhang F, Wang X, et al. (2013): Rare SERINC2 variants are specific for alcohol dependence in individuals of European descent. Pharmacogenet Genomics 23:395402.

25. Zuo L, Zhang X, Deng H-w, Luo X (2013): Association of rare PTP4A1PHF3-EYS variants with alcohol dependence. J Hum Genet 58:178179.

26. Haller G, Li P, Esch C, Hsu S, Goate AM, Steinbach JH (2014): Functional characterization improves associations between rare nonsynonymous variants in CHRNB4 and smoking behavior. PLoS One 9:e96753.

27. Duncan LE, Keller MC (2011): A critical review of the first 10 years of candidate gene-by-environment interaction research in psychiatry. Am J Psychiatry 168:1041-1049.

28. Vrieze SI, Feng S, Miller MB, Hicks BM, Pankratz N, Abecasis GR, et al. (2014): Rare nonsynonymous exonic variants in addiction and behavioral disinhibition. Biol Psychiatry 75:783-789.

29. Vrieze SI, Malone SM, Vaidyanathan U, Kwong A, Kang HM, Zhan X, et al. (2014): In search of rare variants: Preliminary results from whole genome sequencing of 1,325 individuals with psychophysiological endophenotypes. Psychophysiology 51:1309-1320.

30. Vrieze SI, Malone SM, Pankratz N, Vaidyanathan U, Miller MB, Kang HM, et al. (2014): Genetic associations of nonsynonymous exonic variants with psychophysiological endophenotypes. Psychophysiology 51:1300-1308.

31. McCarthy S, Das S, Kretzschmar W, Delaneau O, Wood AR, Teumer A, et al. (2016): A reference panel of 64,976 haplotypes for genotype imputation. Nat Genet 48:1279-1283.

32. Evans LM, Tahmasbi R, Vrieze SI, Abecasis GR, Das S, Gazal S, et al. (2018): Comparison of methods that use whole genome data to estimate the heritability and genetic architecture of complex traits. Nature Genet 50:737-745.

33. Schaid DJ, Chen W, Larson NB (2018): From genome-wide associations to candidate causal variants by statistical fine-mapping. Nat Rev Genet 19:491-504.

34. Hicks BM, Schalet BD, Malone SM, lacono WG, McGue M (2011): Psychometric and genetic architecture of substance use disorder and behavioral disinhibition measures for gene association studies. Behav Genet 41:459-475.

35. Vrieze SI, McGue M, Miller MB, Hicks BM, lacono WG (2013): Three mutually informative ways to understand the genetic relationships among behavioral disinhibition, alcohol use, drug use, nicotine use/ dependence, and their co-occurrence: Twin biometry, GCTA, and genome-wide scoring. Behav Genet 43:97-107.

36. Vink JM, Willemsen G, Boomsma DI (2005): Heritability of smoking initiation and nicotine dependence. Behav Genet 35:397-406.

37. Maes HH, Sullivan PF, Bulik CM, Neale MC, Prescott CA, Eaves LJ, et al. (2004): A twin study of genetic and environmental influences on tobacco initiation, regular tobacco use and nicotine dependence. Psychol Med 34:1251-1261.

38. Swan GE, Carmelli D, Rosenman RH, Fabsitz RR, Christian JC (1990): Smoking and alcohol consumption in adult male twins: Genetic heritability and shared environmental influences. J Subst Abuse 2:39-50.

39. Schumann G, Liu CY, O'Reilly P, Gao H, Song P, Xu B, et al. (2016): KLB is associated with alcohol drinking, and its gene product betaKlotho is necessary for FGF21 regulation of alcohol preference. Proc Natl Acad Sci U S A 113:14372-14377.

40. Jorgenson E, Thai KK, Hoffmann TJ, Sakoda LC, Kvale MN, Banda Y, et al. (2017): Genetic contributors to variation in alcohol consumption vary by race/ethnicity in a large multi-ethnic genome-wide association study. Mol Psychiatry 22:1359-1367.

41. Thorgeirsson TE, Gudbjartsson DF, Surakka I, Vink JM, Amin N, Geller F, et al. (2010): Sequence variants at CHRNB3-CHRNA6 and CYP2A6 affect smoking behavior. Nature Genet 42:448-453.

42. Bycroft C, Freeman C, Petkova D, Band G, Elliott LT, Sharp K, et al. (2017): Genome-wide genetic data on $\sim 500,000$ UK Biobank participants [published online ahead of print Jul 20]. bioRxiv.

43. Kang HM, Sul JH, Service SK, Zaitlen NA, Kong SY, Freimer NB, et al. (2010): Variance component model to account for sample structure in genome-wide association studies. Nat Genet 42:348-354.

44. Liu DJ, Peloso GM, Zhan X, Holmen OL, Zawistowski M, Feng S, et al. (2014): Meta-analysis of gene-level tests for rare variant association. Nat Genet 46:200-204.

45. Pruitt KD, Brown GR, Hiatt SM, Thibaud-Nissen F, Astashyn A, Ermolaeva O, et al. (2014): RefSeq: An update on mammalian reference sequences. Nucleic Acids Res 42:D756-D763.

46. Wu MC, Lee S, Cai TX, Li Y, Boehnke M, Lin XH (2011): Rare-variant association testing for sequencing data with the sequence kernel association test. Am J Hum Genet 89:82-93.

47. Lee S, Wu MC, Lin X (2012): Optimal tests for rare variant effects in sequencing association studies. Biostatistics 13:762-775.

48. Li B, Leal SM (2008): Methods for detecting associations with rare variants for common diseases: Application to analysis of sequence data. Am J Hum Genet 83:311-321.

49. Jiang Y, Chen S, McGuire D, Chen F, Liu M, lacono WG, et al. (2018): Proper conditional analysis in the presence of missing data: Application to large scale meta-analysis of tobacco use phenotypes. PLoS Genet 14:e1007452.

50. Mahajan A, Taliun D, Thurner M, Robertson NR, Torres JM, Rayner NW, et al. (2018): Fine-mapping of an expanded set of type 2 diabetes loci to 


\section{Exome Meta-analysis of Smoking and Alcohol}

single-variant resolution using high-density imputation and isletspecific epigenome maps. Nat Genet 50:1505-1513.

51. Pickrell JK (2014): Joint analysis of functional genomic data and genome-wide association studies of 18 human traits. Am J Hum Genet 94:559-573.

52. Bulik-Sullivan BK, Loh PR, Finucane HK, Ripke S, Yang J, Schizophrenia Working Group of the Psychiatric Genomics Consortium, et al. (2015): LD Score regression distinguishes confounding from polygenicity in genome-wide association studies. Nat Genet 47:291-295.

53. Bulik-Sullivan BK, Loh PR, Finucane HK, Ripke S, Yang J, Patterson N, et al. (2015): LD Score regression distinguishes confounding from polygenicity in genome-wide association studies. Nature Genet 47:291-295.

54. Bulik-Sullivan B, Finucane HK, Anttila V, Gusev A, Day FR, Loh PR, et al. (2015): An atlas of genetic correlations across human diseases and traits. Nature Genet 47:1236-1241.
55. Zhan X, Liu DJ (2015): SEQMINER: An R-package to facilitate the functional interpretation of sequence-based associations. Genet Epidemiol 39:619-623.

56. Lassi G, Taylor AE, Timpson NJ, Kenny PJ, Mather RJ, Eisen T, et al. (2016): The CHRNA5-A3-B4 gene cluster and smoking: From discovery to therapeutics. Trends Neurosci 39:851-861.

57. Edenberg HJ (2007): The genetics of alcohol metabolism: Role of alcohol dehydrogenase and aldehyde dehydrogenase variants. Alcohol Res Health 30:5-13.

58. Ghitza UE, Zhai H, Wu P, Airavaara M, Shaham Y, Lu L (2010): Role of BDNF and GDNF in drug reward and relapse: A review. Neurosci Biobehav Rev 35:157-171.

59. Paul S, Amundson SA (2014): Differential effect of active smoking on gene expression in male and female smokers. J Carcinog Mutagen 5:100198. 